\title{
Joint toxicity of fluoroquinolone and tetracycline antibiotics to zebrafish (Danio rerio) based on biochemical biomarkers and histopathological observation
}

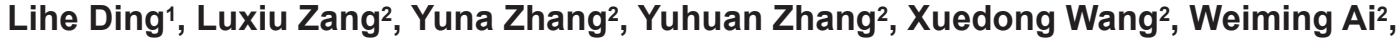 \\ Nani Ding ${ }^{2, *}$ and Huili Wang2,\# \\ ${ }^{I}$ School of Physical Education and Sport Sciences, Wenzhou Medical University, Wenzhou 325035, China \\ ${ }^{2}$ College of Life Sciences, Wenzhou Medical University, Wenzhou 325035, China
}

(Received September 30, 2016; Accepted January 23, 2017)

\begin{abstract}
Herein, we report on the joint toxicity of four fluoroquinolones and two tetracyclines ( $\beta$-diketone antibiotics-DKAs) to zebrafish based on a series of toxicological endpoints and histopathological observations. A positive dose-dependence was observed in DKA-exposure groups with a 72-hpf

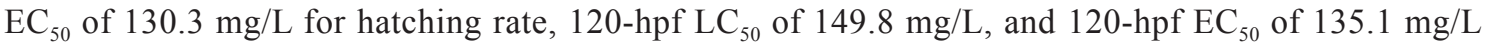
for malformation rate. When zebrafish at $60 \mathrm{dpf}$ were exposed to a series of DKA concentrations (45, 60 and $90 \mathrm{mg} / \mathrm{L}$ ) for 7, 14 and 21 days, creatine kinase and $\mathrm{AChE}$ activities were significantly induced, and intracellular malondialdehyde increased in all treatments except for the $45 \mathrm{mg} / \mathrm{L}$ treatment. The transcription levels of AHRRa from livers were significantly $(p<0.05)$ up-regulated in all treatments after two months of DKA exposure. CKma expression from skeletal muscle was significantly down-regulated in the $90 \mathrm{mg} / \mathrm{L}$ treatment. A remarkable down-regulation of CYP $3 A 65$ was observed in the $60 \mathrm{mg} / \mathrm{L}$ treatment. DKA exposure resulted in severe tissue damage including mitochondria swelling, reduction of mitochondrial cristae, deepening of mitochondrial cristae bands, and decreasing and even disappearance of the rough endoplasmic reticulum. Total sperm motility was decreased by ca. $30 \%$ due to DKA exposure. These results provide important information for toxicity and health risks due to mixed DKA exposure in aquatic environments.
\end{abstract}

Key words: $\beta$-diketone antibiotics, Creatine kinase, AChE activities, Intracellular malondialdehyde, Expression of CYP3A65, AHRRa and CKma, Sperm motility

\section{INTRODUCTION}

Fluoroquinolones (FQs) and tetracyclines (TCs) are two representative families of broad-spectrum antibiotics that are widely used to prevent and treat a large variety of infectious diseases in humans and veterinary practices (Lanza and Sellergren, 1999). They are named $\beta$-diketone antibiotics (DKAs) due to the presence of a diketone group in their molecular structure ( $\mathrm{Qu}$ et al., 2010). Aquaculture operations have especially raised environmental concerns due to the heavy and diverse use of antibiotics and their potential impacts on downstream aquatic ecosystems (Bondad-Reantaso et al., 2012; Rico et al., 2012). For example, over 8,000 ton of DKAs are used as feed additives in China each year (Ben et al., 2008). Qui- nolones and their fluoro derivatives, such as ofloxacin or ciprofloxacin, are widely used as antimicrobial agents for their broad spectrum activity and favorable pharmacokinetics. Because DKAs are weakly absorbed and not completely metabolized in the animal's gut, the majority of DKAs are excreted intact in feces and urine (Shi et al., 2012). Although the recorded environmental concentrations of FQs and TCs are usually low, at $\mathrm{ng} / \mathrm{L}$ to $\mathrm{mg} / \mathrm{L}$ levels in waters and $\mu \mathrm{g} / \mathrm{kg}$ to $\mathrm{mg} / \mathrm{kg}$ levels in soils and sediments, they are considered "pseudopersistant" contaminants due to their continued release into the environment and permanent presence. In hospital sewage water, large temporal variations in FQ and TC concentrations were observed in the range of 3.6-101.0 $\mathrm{mg} / \mathrm{L}$ for ciprofloxacin, 0.2-7.6 mg/L for ofloxacin, and 0.6-6.7 mg/L for

*Co-corresponding: Nani Ding (E-mail: dnn@wzmc.edu.cn)

* Corresponding: Huili Wang (E-mail: whuili@163.com) 
L. Ding et al.

doxycycline (Lindberg et al., 2004). In Turkey, oxytetracycline was detected in agricultural soils receiving animal manure at a maximum concentration of $0.5 \mathrm{mg} / \mathrm{kg}$ (Karci and Balcioğlu, 2009). Several studies conducted in Beijing and Tianjin suburbs of China reported tetracycline concentrations as high as $119-307 \mathrm{mg} / \mathrm{kg}$ in agricultural soils irrigated with domestic wastewater and pig feces (Xie et al., 2011).

The continuous application of FQs and TCs poses a great threat to ecological and human health due to their adverse effects on aquatic systems. For example, amoxicillin and oxytetracycline were reported to have high potential risk for aquatic environments in England and Korea (Jones et al., 2002; Lee et al., 2008). Tetracyclines facilitate a resultant fatty liver by affecting the gene expression associated with lipid metabolism for increasing triglyceride (TG) and cholesterol biosynthesis and decreasing $\beta$-oxidation of fatty acids (Yin et al., 2006). Previous studies showed that DKAs have strong genetic toxicity resulting in DNA damage, adduct formation, DNA hypermethylation of kidney cells in Carassius auratus and teratogenic effects on Cyprinus carpio (Khadra et al., 2012; Li et al., 2010). Among the toxicities of quinolones, one major risk is their chondrotoxicity potential in juvenile animals, such as rats, dogs, rabbits, nonhuman primates, and others (Sheng et al., 2013). Recent observations have also shown that intracellular $\mathrm{H}_{2} \mathrm{O}_{2}$ is markedly increased after exposure to $10 \mu \mathrm{g} / \mathrm{mL}$ ofloxacin for $12 \mathrm{hr}$ in monolayer cultured chondrocytes (Li et al., 2010). Schwartz and co-workers found that minocycline and doxycycline could depolarize mitochondria at a high concentration and inhibit matrix metalloprotease (Schwartz et al., 2013). Zhang and coworkers reported that the $96-\mathrm{hr} \mathrm{LC}_{50}$ values of tetracycline were 406.0 and $322.8 \mathrm{mg} / \mathrm{L}$ in zebrafish and carp, respectively (Zhang et al., 2008). Tetracycline and chlortetracycline induced significant genotoxicity on earthworm Eisenia fetida in a dose-dependent manner with chlortetracycline having a stronger effect than tetracycline in short-term exposure (Dong et al., 2012). TCs could significantly inhibit the growth and physiological progresses including primary photochemistry and the antioxidant system (Yang et al., 2013). Long-term exposure to low-dose DKA mixtures can induce behavioral, biomarker and histopathological processes leading to the origin of human diseases (Scott and Sloman, 2004). These toxicological endpoints based on zebrafish (Danio rerio) provide valuable information about DKA toxicity and safety in human health.

Zebrafish have become a preferred toxicity model due to their rapid life cycle, high fecundity, transparent devel- opment, and their embryos being amenable to genetic manipulation using transgenic approaches and morpholino gene knockdowns (Sipes et al., 2011). Another important attribute is that sequencing of the zebrafish genome has shown more than $80 \%$ of zebrafish genes are homologous to human genes. As a result, conservation and homology between human and zebrafish allow for effective modeling of human diseases (Allen and Neely, 2010).

Previous reports mainly focused on toxicity and associated mechanisms for a single DKA compound (Khadra et al., 2012; Li et al., 2010; Wang et al., 2014). However, little information is available on the aquatic toxicity of mixed DKAs using zebrafish (Danio rerio) as a model organism. In real-world environments, hundreds of DKAs are often detected in environmental samples (Lindberg et al., 2004; Shi et al., 2012), and thus they may interact with each other resulting in changes in their combined toxicity potential. Toxicological tests for single and mixed DKA species are both important, because these data reflect real-world environmental concentrations and the interactive relationship between single species. For example, while a single DKA compound in an aquatic environment may not induce any acute toxicity effect, the interaction among DKAs in mixtures may lead to significant toxicity to aquatic organisms. The combined effects of DKAs may change with composition of mixtures, exposure duration, receptor sensitive period, pathway, etc. For in vivo experiments, the changes in metabolic transformation and distribution of DKAs make toxicological research more complicated. Therefore, the mixed toxicological effect of DKAs is a challenging and practical subject in real-world aquatic environments.

In this study, we chose four FQs and two TCs (equal mass proportion), based on their frequent detection in aquatic environments, as representative DKA species to investigate their joint toxicological effects on zebrafish (Danio rerio). Several toxicological endpoints were examined at the zebrafish embryo-larval stage, which included malformation, hatching, mortality, creatine kinease (CK) activity, creatinine $(\mathrm{Cr})$ concentration, acetylcholine esterase $(\mathrm{AChE})$ activity, and concentration of the oxidative product malondialdehyde (MDA). In addition, the transcriptional levels of CYP3A65 (cytochrome P450, family 3, subfamily A, polypeptide 65), AHRRa (aryl-hydrocarbon receptor repressor a), and CKma (creatine kinase, muscle a) involved in skeletal muscle tissue were examined using qRT-PCR with adult zebrafish after two months of DKA exposure. Histopathological analysis of liver and skeletal muscle was performed to detect evidence of tissue damage. Results of this study provide important information for toxicity and health risks resulting from exposure to 
Toxicity of $\beta$-diketone antibiotics to zebrafish

mixed DKAs in aquatic environments.

\section{MATERIALS AND METHODS}

\section{Chemicals}

Four FQs (ciprofloxacin, ofluoxacin, norfloxacin, and enrofloxacin) and two TCs (chlortetracycline and doxycycline) were used in this study. Their purities and $\mathrm{pKa}$ values are listed in Supplementary Table 1. The six species of DKAs were all sourced from Amresco (AR, Solon, $\mathrm{OH}, \mathrm{USA}$ ) and used as received.

\section{Fish husbandry}

Wild-type zebrafish (Danio rerio, AB strain) were raised in an aquarium receiving circulated and aerated tap water at $28^{\circ} \mathrm{C}$ with a $14: 10$-hr light/dark cycle (lights on at 8:00 a.m.) according to standard zebrafish breeding protocols (Westerfield, 2000). Water supplied to the system was dechlorinated and filtered by reverse osmosis ( $\mathrm{pH}$ 6.5-7.5, ammonia $<0.01 \mathrm{mg} \mathrm{L}^{-1}$ and dissolved oxygen $\geq 95 \%$ saturation), and instant ocean salt was added to raise the conductivity to $450-1000 \mu \mathrm{S} / \mathrm{cm}$. Zebrafish were fed twice daily with live Artemia (Jiahong Feed Co., Tianjin, China) and dry flake diet (Zeigler, Aquatic Habitats, Apopka, FL, USA). Zebrafish embryos were obtained from spawning adults in tanks overnight with a sex ratio of $1: 1$. Animal stages were recorded as hours post fertilization (hpf) or days post fertilization (dpf). DKA-exposure intervals were recorded as days after exposure (DAE). Prior to DKA exposure, normal embryos were incubated in Petri dishes at $28 \pm 0.5^{\circ} \mathrm{C}$ with unfertilized and abnormal embryos removed under a stereomicroscope.

\section{Exposure protocols}

Based on previous toxicity information for these DKA compounds, we conducted preliminary experiments to determine exposure concentrations for each toxicological endpoint (Wang et al., 2014). According to the results, we selected $0,18.75,37.5,75,150,300$ and $450 \mathrm{mg} / \mathrm{L}$ as exposure concentrations (the concentration for each DKA species was $0,3.13,6.25,12.50,25.00,50.00$ and $75.00 \mathrm{mg} / \mathrm{L}$ ) for the determination of $\mathrm{EC}_{50}$ for hatching rate, $\mathrm{LC}_{50}$ and $\mathrm{EC}_{50}$ for malformation rate, and the exposure period was set in the range of 6 to $120 \mathrm{hpf}$. Zebrafish at $60 \mathrm{dpf}$ were exposed to DKAs at a series of concentrations $(0,45,60$ and $90 \mathrm{mg} / \mathrm{L})$. At 7, 14 and 21 daysafter-exposure, 10 zebrafish were collected for the determination of $\mathrm{CK}$ and AchE activities, and $\mathrm{Cr}$ and MDA concentrations. Phenylthiourea $(0.1 \mathrm{mM})$ was added to embryonic medium to increase the transparency of zebrafish larvae, and the heart rhythms and circulation were observed without the need for intervention (Craig et al., 2006). Phenylthiourea is frequently used in zebrafish morphological observations to block melanin synthesis and prevent pigmentation of developing embryos. The embryos incubated in $0.003 \%(0.2 \mathrm{mM})$ phenylthiourea did not produce any morphological defects except missing pigment (Westerfield, 2000). In this study, we set $0.1 \mathrm{mM}$ phenylthiourea as the control and verified that it had no effect on hatching and malformation rates at 120 hpf (data not shown). The control group was incubated in aerated tap water without addition of DKAs. All of the experiments were performed at $28^{\circ} \mathrm{C}$ in a light-controlled incubator. During the experiment, saturated oxygen was maintained in the solution. Three biological replicates and three technological replicates were conducted for each control and DKA-exposure treatment group. Each tank was equipped with an air pump and periodically cleared of fecal residue and dead fish. Feeding of the zebrafish was stopped two days before harvesting for subsequent analyses.

The DKA stock solution was prepared by dissolving the six DKA species in ultra-pure water using $0.1 \mathrm{~mol} / \mathrm{L}$ $\mathrm{NaOH}$ as co-solvent. DKA-exposure treatments were conducted in tap water by fortifying with an appropriate amount of DKA stock solution, which was adjusted to $\mathrm{pH}$ 7.2-7.3 with $\mathrm{NaOH}$. In order to maintain constant DKA concentrations for the period of exposure, DKA solutions were renewed with freshly prepared solution each day. To assure accuracy and reproducibility, a minimum survival rate of $95 \%$ was set for the control group.

\section{Preparation of biological samples}

Biochemical activity or concentration was determined in isolated tissues from adults for each 7-day period beginning two weeks after exposure. After DKA exposure, 10 zebrafish for each biological replicate were anesthetized with $0.03 \%$ tricaine (MS-222). Triplicate biological treatments were performed using a total of 30 zebrafish at $90 \mathrm{dpf}$, and their lengths and weights were recorded. Peripheral blood was collected from the caudal vein of each fish followed by addition of heparin to prevent clotting of the blood. The blood samples were centrifuged at $6.2 \mathrm{~g}$ for $10 \mathrm{~min}$ at $4^{\circ} \mathrm{C}$, and the blood serum was collected and stored at $-80^{\circ} \mathrm{C}$ for subsequent bioassay. Concurrently, the skeletal muscle, liver and whole brain were dissected over ice, weighed, and homogenized in 9-fold volumes of ice-cold $\mathrm{NaCl}(0.9 \%)$ solution in a hand-held glass homogenizer. The homogenated mixture was centrifuged at $13.8 \mathrm{~g}$ for $10 \mathrm{~min}$ at $4^{\circ} \mathrm{C}$, and the resulting supernatant was used to test enzymatic activity or stored sepa- 
rately at $-80^{\circ} \mathrm{C}$ for biochemical assay.

\section{Determination of biomarkers}

Zebrafish at $60 \mathrm{dpf}$ were exposed to different concentrations of DKAs $(0,45,60$ and $90 \mathrm{mg} / \mathrm{L})$, and 10 zebrafish for each group were collected at exposure intervals of 7, 14 and 21 days, and pretreated as described above for the determination of biomarkers. CK activity $(\mathrm{U} / \mathrm{mg}$ prot$)$ and $\mathrm{Cr}(\mu \mathrm{mol} / \mathrm{L})$ concentration from zebrafish muscle were determined using test kits following manufacturer's specification (Nanjing Jiancheng Bioengineering Institute, Nanjing, China). The MDA (nmol/mg prot) content from zebrafish liver was determined by thiobarbituric acid (TBA) colorimetry (Li et al., 2009). AChE activity (U/mg prot) from zebrafish brain was analyzed using a commercial AChE detection kit (Guangdong Dongsheng, Guangzhou, China) according to manufacturer's instructions and a previous publication (Jansen et al., 2009). Enzymatic activities were determined in triplicate and expressed as nanomoles of substrate hydrolyzed per minute per mg of protein. Soluble protein concentrations were measured using an Enhanced BCA Protein Assay Kit (Beyotime Institute of Biotechnology, Nanjing, China) (Ye et al., 2008).

\section{qRT-PCR analysis of candidate genes}

Three candidate genes (CYP3A65, AHRRa from liver and CKma from skeletal muscle) were chosen for verification of changes in their transcriptional level using qRTPCR. Candidate gene sequences were obtained from the zebrafish genome (Danio rerio, version zv 9.0) (http:// asia.ensembl.org/Danio_rerio/Info/Index). qRT-PCR was performed using the StepOne ${ }^{\mathrm{TM}}$ RT-PCR System (Applied Biosystems, Foster, CA, USA). Zebrafish at $60 \mathrm{dpf}$ were exposed to a series of DKA concentrations $(0,45,60$ and $90 \mathrm{mg} / \mathrm{L}$ ) for two months prior to extraction of total RNA. The quality of RNA was measured by the Nanodrop method with minimum requirements of $\mathrm{OD}_{260} / \mathrm{OD}_{280}>1.8$ and $\mathrm{OD}_{260} / \mathrm{OD}_{230}>1.5$. After the RNA was detected without protein and salt contamination, cDNA was obtained by reverse transcription using a PrimeScript RT-PCR Kit (TaKaRa, Shiga, Japan). Primer sets were designed using Premier 5.0 software and synthesized by Shanghai Sangon Biotechnology Co. (Shanghai, China). The primer pairs were designed to span introns to prevent amplification of any contaminating genomic DNA. The details of these fragments are summarized in Supplementary Table 2. Each sample in each group was measured in triplicate and each experiment was replicated at least three times. $\beta$-actin was used as a normalization control and the control served as the reference sample. The comparative $\mathrm{Ct}$ method was used for calculating the relative expression levels of mRNA.

\section{Preparation of biological samples for transmission electron microscopy (TEM)}

Ten zebrafish at $60 \mathrm{dpf}$ were exposed to DKAs at a series of concentrations $(0,45,60$ and $90 \mathrm{mg} / \mathrm{L})$ for two months, and then collected, washed with distilled water to remove surface dunghills and surface dried with filter paper. Histopathological evidence of tissue damage was examined using TEM (TEM 10; Zeiss, Jena, Germany). The liver and skeletal muscle were dissected in control and treatment groups, cut into $1 \mathrm{~mm}^{3}$ tissue blocks and fixed in glutaraldehyde at $4^{\circ} \mathrm{C}$. A series of pretreatment procedures, such as poaching, fixing, dehydrating, embedding and sectioning, were performed to prepare tissues for TEM observation according to Sendzik and coworkers (Sendzik et al., 2010).

\section{Determination of total sperm motility}

When zebrafish at $60 \mathrm{dpf}$ were exposed to a series of DKA concentrations $(0,45,60$ and $90 \mathrm{mg} / \mathrm{L})$ for two months, the total sperm motility was determined according to Martyniuk and co-workers (Martyniuk et al., 2007). Briefly, 10 male zebrafish were randomly selected from each treatment, anesthetized for $2 \mathrm{~min}$ in $0.01 \%$ tricaine methane sulfonate (MS-222, Western Chemical Inc., Ferndale, WA, USA), rinsed in distilled water, and blotted dry with a paper towel. Before dissection, standard length (from snout tip to the base of the caudal fin) and body weight were measured. The testes were removed and separated from surrounding lipid tissues using a dissecting microscope (10× magnification), and transferred to $1.5-\mathrm{mL}$ centrifuge tubes for weighing. Hank's balanced salt solution (HBSS) at an osmolality of $300 \mathrm{mosmol} / \mathrm{kg}$ (HBSS300: $0.137 \mathrm{M} \mathrm{NaCl}, 5.4 \mathrm{mM} \mathrm{KCl}, 1.3 \mathrm{mM} \mathrm{CaCl}_{2}$,

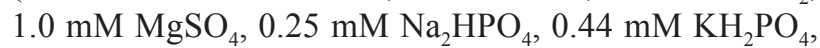
$4.2 \mathrm{mM} \mathrm{NaHCO}_{3}, 5.55 \mathrm{mM}$ glucose, $\mathrm{pH}$ 7.2) was used as extender unless other osmolalities were prepared in experiments by adjusting water volume. Hereafter, HBSS with a specific osmolality, such as $300 \mathrm{mosmol} / \mathrm{kg}$, was abbreviated as "HBSS300". Sperm was suspended by gently crushing the testes in HBSS and sperm suspensions were held on ice until used. The sperm sample $(5 \mu \mathrm{L})$ after removal of impurities was activated by $5 \mu \mathrm{L}$ ultrapure water and then injected to a specific glass hole (Leja, Luzernestraat, Nieuw-Vennep, Netherland). The sperm motility and concentration were determined under a Sperm Motility Analyzer (IVOS, Beverly, MA, USA). The motility was expressed as the percentage of sperm which actively moved in a forward direction; sperm that vibrated in 
Toxicity of $\beta$-diketone antibiotics to zebrafish

place was not counted as motile. Each sample was analyzed at least two times in three microscopic fields.

\section{Statistical analysis}

Each treatment and control group was performed with 3 biological replicates and 3 technological replicates, and all data were recorded as mean \pm S.D. (standard deviation, $\mathrm{n}=3$ ). The concentration-response curves used to determine $\mathrm{EC}_{50}$ values were computed using Origin 8.0 Software (OriginLab, Northampton, MA, USA). Oneway ANOVA was performed to calculate statistical significance followed by post-hoc Dunnett's or Tukey's tests to independently compare each DKA-exposure group to the control group. All statistical analyses were conducted with SPSS 18.0 (SPSS, Chicago, IL, USA) using a $p<$ 0.05 or $p<0.01$ significance level.

\section{RESULTS}

\section{Effects of mixed DKAs on zebrafish hatching and mortality rates}

According to our previous results, the joint toxicity of
FQs and TCs to zebrafish was comparable with or slightly less than those of TCs alone, and thus TCs played a key toxicological role in the combined DKAs (Wang et al., 2014). Based on analysis from three assessment methods (Toxic Unit, Additional Index and Mixture Toxic Index), the interaction between FQs and TCs was an antagonistic effect. However in real-world environments, FQs and TCs co-exist in aquatic ecosystems. Because the four FQs (ciprofloxacin, ofluoxacin, norfloxacin, and enrofloxacin) and two TCs (chlortetracycline and doxycycline) are detected with high frequency in aquatic environments, they were selected to investigate their joint effects on larval zebrafish hatching and mortality rates. When embryos were exposed to DKA mixtures, there was an obvious concentration-dependent decrease in the 72-hpf hatching rate at concentrations ranging from 75 to $450 \mathrm{mg} / \mathrm{L}$ (Fig. 1A). No difference was observed between the control and the 18.5 or $37.5 \mathrm{mg} / \mathrm{L}$ DKA treatments, while significant differences $(p<0.05$ or $p<0.01)$ for hatching rates were observed at DKA exposures $>75 \mathrm{mg} / \mathrm{L}$. When embryos were exposed to DKA concentrations $>150 \mathrm{mg} / \mathrm{L}$, no hatching was observed by the end of
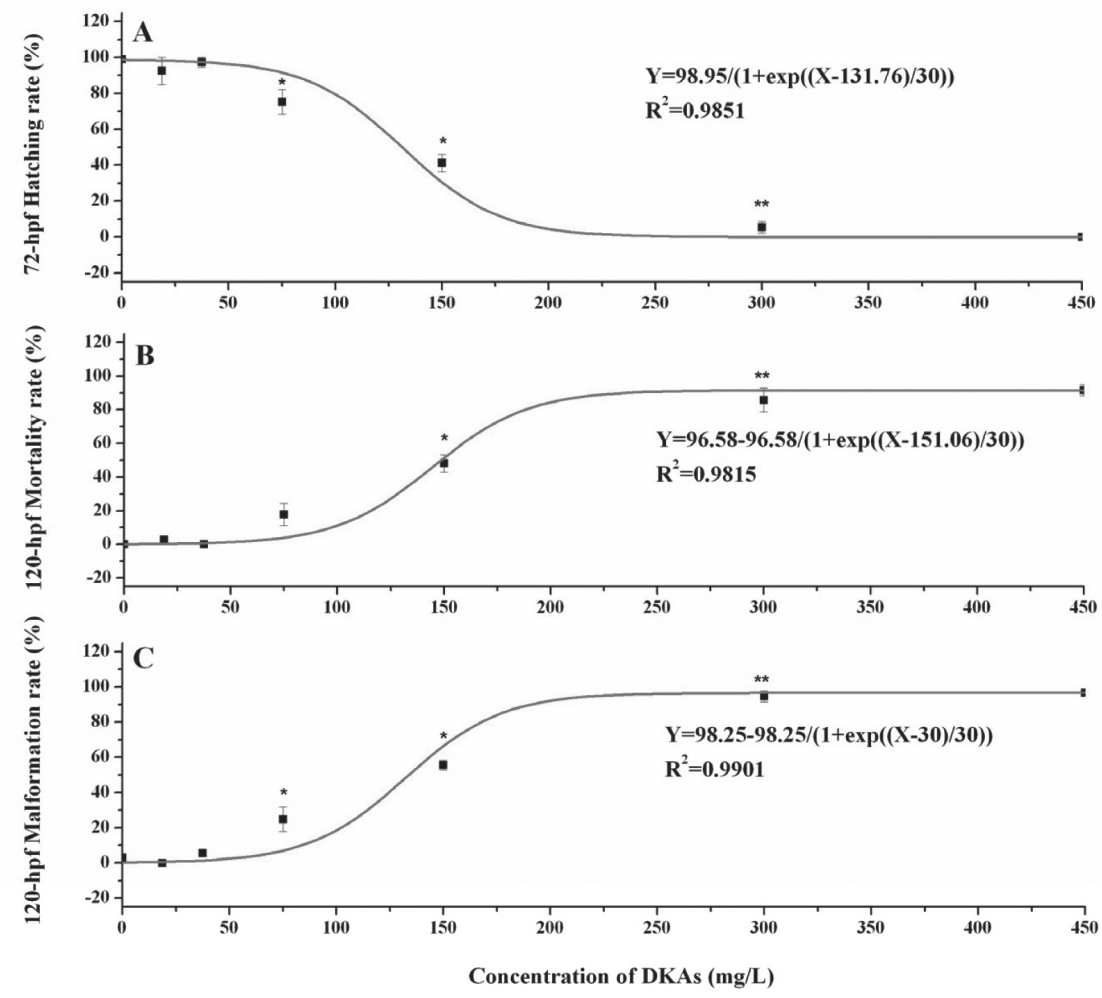

Fig. 1. Effects of DKA exposure concentrations on zebrafish development. (1) A, on hatch rate at 72 hpf ( $\mathrm{n}=30$ embryos); (2) B, on mortality at $120 \mathrm{hpf}(\mathrm{n}=30$ embryos); (3) C, on malformation at $120 \mathrm{hpf}(\mathrm{n}=30$ embryos $) ;(4) *$ and ** indicate significant difference from control at $p<0.05$ and $p<0.01$ levels, respectively; (5) Values represent the average \pm S.D. $(\mathrm{n}=3$ ). 
the experiment (120 hpf). The resulting 72-hpf $\mathrm{EC}_{50}$ for hatching rate in the DKA mixture was calculated to be $130.3 \mathrm{mg} / \mathrm{L}$. In comparison, it was $13.8 \mathrm{mg} / \mathrm{L}$ with TC exposure alone (Supplementary Table 3 ).

A positive dose-dependence mortality rate for the DKA-exposure groups was observed at $120 \mathrm{hpf}$ (Fig. 1B). No zebrafish mortality was recorded for the control, 18.5 or $37.5 \mathrm{mg} / \mathrm{L}$ DKA mixtures (corresponding to 0, 3.1, and $6.25 \mathrm{mg} / \mathrm{L}$ for each of the six DKA species), and the survival rates were $>95 \%$ at $120 \mathrm{hpf}$. In contrast, there was a dramatic increase of the mortality rate between DKA concentrations of 75 and $300 \mathrm{mg} / \mathrm{L}$ (Fig. 1B). For example, the mortality rate was $>51 \%$ for the $150 \mathrm{mg} / \mathrm{L} \mathrm{DKA}$ treatment, while all zebrafish died in the $300 \mathrm{mg} / \mathrm{L}$ DKA treatment. As a result, the $\mathrm{LC}_{50}$ of DKAs at $120 \mathrm{hpf}$ was computed to be $149.8 \mathrm{mg} / \mathrm{L}$. In contrast, the $\mathrm{LC}_{50}$ of TCs at $120 \mathrm{hpf}$ was $18.3 \mathrm{mg} / \mathrm{L}$, suggesting that TCs were more toxic than the mixed DKAs (4 FQs and 2 TCs as listed in Supplementary Table 3).

\section{Zebrafish malformation by DKA exposure}

Exposure of zebrafish embryos-larva to mixed DKAs led to lethal as well as various nonlethal malformations. Zebrafish were exposed to DKAs from 6 to $120 \mathrm{hpf}$, and malformations were recorded at $120 \mathrm{hpf}$ (Fig. 1C). The 120-hpf $\mathrm{EC}_{50}$ for malformation rate was calculated to be $135.1 \mathrm{mg} / \mathrm{L}$ (corresponding to $22.5 \mathrm{mg} / \mathrm{L}$ for each of the six DKA species). In comparison, the 120-hpf $\mathrm{EC}_{50}$ values for malformation rate due to TCs and FQs-exposure were 16.4 and $481.3 \mathrm{mg} / \mathrm{L}$, respectively (Supplementary Fig. 1).

In the control group, no malformation phenomena such as hatching delay, uninflated swim bladder and crooked body were observed, while severe malformations occurred in DKA treatments. Because the analysis of embryolarval toxicological results is based on an estimation of the frequency and intensity of abnormalities observed, the selection of endpoints becomes an important criteria. In this study, after DKA exposure several malformation patterns (i.e., hatching delay, uninflated swim bladder (Fig. 2A-D), yolk sac edema (Fig. 2E-H), pericardial edema (Fig. 2M-N) and crooked body (Fig. 2I-L)) were prominent in most treatments (Fig. 2). Among malformations hatching delay (Fig. 2P), uninflated swim bladder and yolk sac edema were considered important malformations because of a relatively higher probability of occurrence. For instance, the embryos subjected to DKA exposure showed yolk sac edema and conjugation (Fig. 2E-H). Higher DKA exposure concentration $(90 \mathrm{mg} / \mathrm{L})$ resulted in yolk sac edema, uninflated swim bladder and pericardial edema (Fig. 2N). Moreover, we found that after DKA exposure to zebrafish embryos and larvae, the otic vesicle produced abnormal growth, and the volume of otolith were prominently reduced with increasing DKA concentrations. In the control group, the utricular and saccular morphologies were very clear and distinguishable, but in the DKA-exposed treatments, the otic vesicle area was obviously decreased (Fig. 2E-H), demonstrating that the amount of hair cells was decreased with increasing DKA concentrations.

\section{Biomarker assay of chronic toxicity}

$\mathrm{CK}$ and $\mathrm{Cr}$, which are related to muscle contraction and energy transfer, were measured as biomarkers to DKA exposure. The Cr/phosphocreatine ( $\mathrm{PCr}$ )/CK system is one of the most important mechanisms for supplying energy in vertebrates. When demanding a higher energy supplement, a more efficient creatine kinase system is required to catalyze phosphocreatine to creatine and ADP to ATP. The $\mathrm{Cr} / \mathrm{PCr}$ system is finely regulated by $\mathrm{CK}$ and is crucial in meeting the cellular energy demand.

In this investigation, $\mathrm{CK}$ activity and $\mathrm{Cr}$ concentration were determined in zebrafish following exposure to a series of DKA concentrations $(45,60$ and $90 \mathrm{mg} / \mathrm{L})$. The increasing trend of $\mathrm{CK}$ activity in response to DKA exposure was similar to locomotor activity (Fig. 3A). The control group demonstrated a decreasing trend in CK activity over time. CK activity was slightly increased 7 days after exposure (DAE) in the 45 and $60 \mathrm{mg} / \mathrm{L}$ treatments. With a further increase in DKA concentration to $90 \mathrm{mg} / \mathrm{L}, \mathrm{CK}$ activity was significantly increased $(p<0.05)$ as compared to the control. In contrast, $\mathrm{CK}$ activities were sharply increased $(p<0.05$ or $p<0.01)$ in all treatments at 14 and 21 DAE in comparison with the control (Fig. 3A), while there was no significant difference between the 45 and $60 \mathrm{mg} / \mathrm{L}$ DKA exposure groups.

$\mathrm{Cr}$ loading has been shown to increase PCr stores, which aids in the rapid production and regeneration of adenosine triphosphate (ATP), and can elicit improvements in high-intensity anaerobic activities (Smith-Ryan et al., 2014). A general decreasing trend in $\mathrm{Cr}$ concentration was observed for all DKA-exposure groups when compared to the control (Fig. 3B). For the $45 \mathrm{mg} / \mathrm{L}$ treatment, $\mathrm{Cr}$ concentration at 7 and 14 DAE was not significantly different $(p>0.05)$ from the control, while it was significantly different at 21 DAE, accounting for $80.3 \%$ of the control group concentration. The most evident reduction in $\mathrm{Cr}$ concentration occurred at 21 DAE, with an average reduction in the 60 and $90 \mathrm{mg} / \mathrm{L}$ DKA treatments of ca. $35-44 \%$ as compared to the control.

DKA-induced changes in AChE activity were detected using whole brain specimens from the zebrafish. 


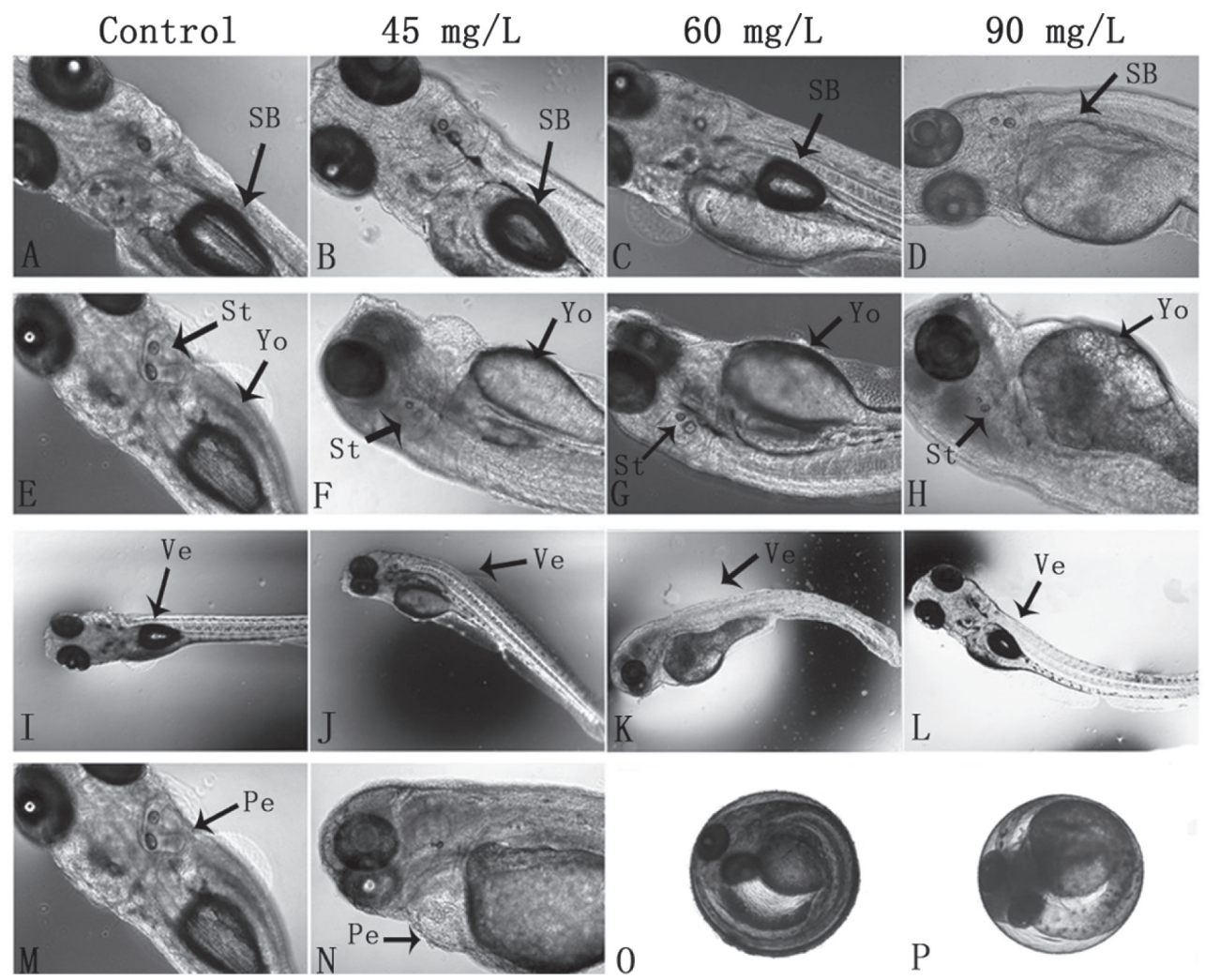

Fig. 2. Malformation profiling of zebrafish after exposure to DKAs at $120 \mathrm{hpf}$. (1) A, E, I and M represent normal larvae; (2) B, F, $\mathrm{J}$ and $\mathrm{N}$ represent $45 \mathrm{mg} / \mathrm{L}$ DKA-exposed treatment; (3) C, G and K represent $60 \mathrm{mg} / \mathrm{L}$ DKA-exposed treatment; (4) D, H, and L represent $90 \mathrm{mg} / \mathrm{L}$ DKA-exposed treatment; (5) SB, swimming bladder; (6) Yo, yolk; (7) St, statocyst; (8) Ve, vertebrae; (9) Pe, pericardium; (10) B-D, swimming bladder gradually becomes small and closed; (11) F-H, malformations of yolk sac edema and statocyst; (12) J-L, body crooked; (13) N, pericardial edema; (14) O, normal embryo; (15) P, unhatched embryo.

AChE activities in brain tissues remained nearly constant in the control group, but were significantly increased in DKA-exposure groups during the experimental intervals $(p<0.01$ or $p<0.05)$ (Fig. 3C). As compared with the control, AChE activity increased by ca. 2-fold at 7, 14 and 21 DAE in DKA-exposure groups $(p<0.01$ for 45 and $90 \mathrm{mg} / \mathrm{L}$ treatments and $p<0.05$ for $60 \mathrm{mg} / \mathrm{L}$ treatment) (Fig. 3C). However, no significant differences in brain AChE activities were observed among treatments at different DKA-exposure intervals (7, 14 and 21 days).

When zebrafish are exposed to adversity, such as oxygen free radicals causing peroxidation of lipids, MDA is formed as an end-product of these oxidation reactions. Thus the concentration of MDA can be used to assess the degree of oxidative damage to lipid peroxidation and the membrane system. MDA content was significantly elevated by DKA exposure in comparison with the control group $(p<0.05)$ and showed a concentration dependence
(Fig. 3D). MDA content was found to be highest at 7 DAE and progressively declined at 14 and 21 DAE, demonstrating that the degree of lipid peroxidation decreased with prolonged DKA exposure. At 7 DAE, MDA content increased approximately $58 \%$ and $71 \%$ for the 60 and $90 \mathrm{mg} / \mathrm{L}$ DKA treatments, respectively. At 14 DAE, only the $90 \mathrm{mg} / \mathrm{L}$ DKA treatment showed a significant difference $(p<0.05)$ relative to the control. At 21 DAE, MDA content in the $90 \mathrm{mg} / \mathrm{L}$ treatment was still significantly higher than that in the control group (Fig. 3D).

\section{Changes in transcriptional levels of $A H R R a$, CYP3A65 and CKma genes}

Long-term DKA exposure can lead to severe disorders in zebrafish based on morphological changes, biomarkers, histopathological analysis and transcriptional level for some related genes. DKA exposure might alter enzyme activities in zebrafish and induce expression of 
L. Ding et al.
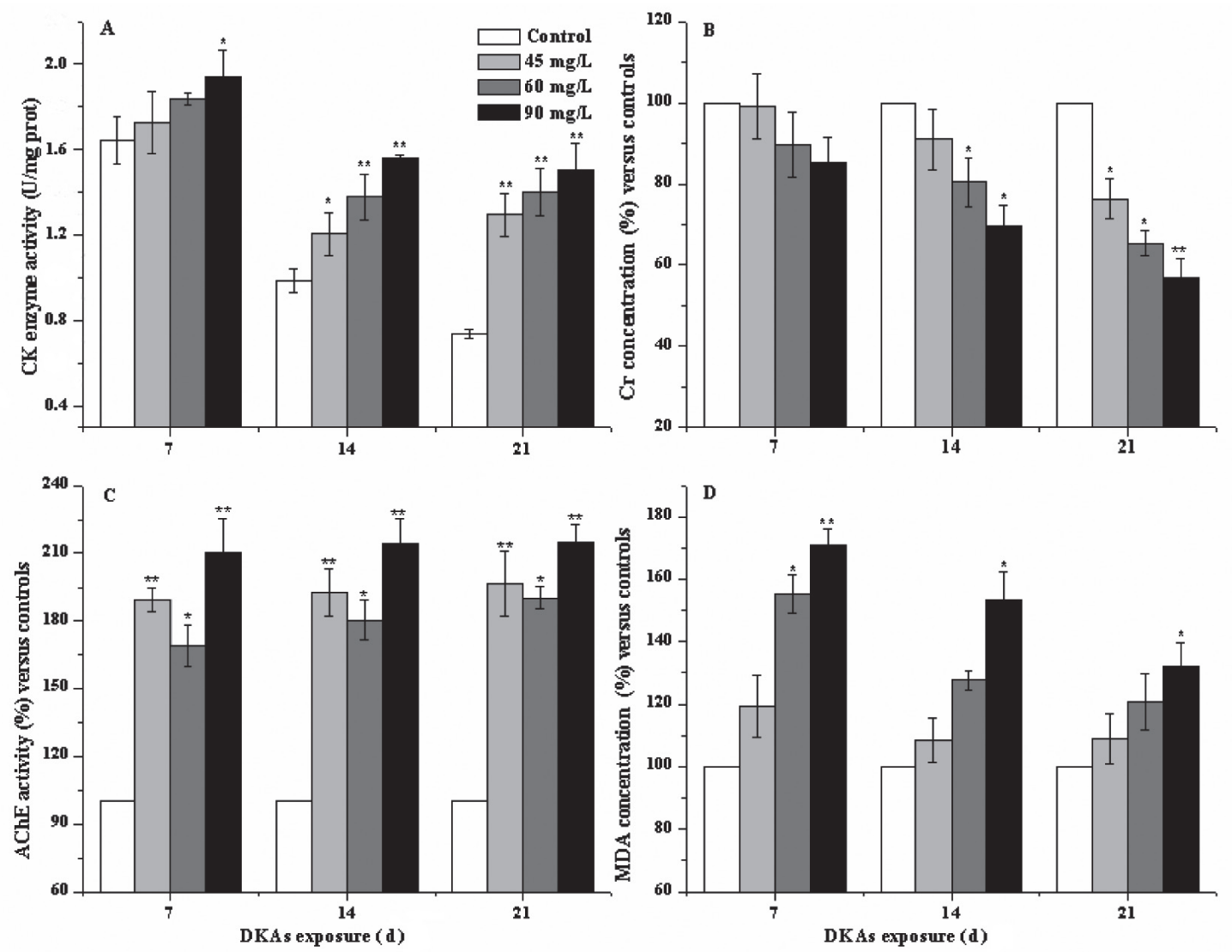

Fig. 3. Effects of DKA concentrations on biomarkers. (1) A, CK activities; (2) B, Cr concentrations; (3) C, AChE activities of zebrafish brain; (4) D, MDA concentrations; (5) Zebrafish at $60 \mathrm{dpf}$ were used for DKA exposure; (6) * and ** indicate significant difference from control at $p<0.05$ and $p<0.01$ levels, respectively; (7) Values represent the average \pm S.D. $(\mathrm{n}=3)$.

genes involved in metabolism, such as transcriptional levels of CYP3A65, AHRRa and CKma. qRT-PCR was used to quantify transcriptional levels for AHRRa, CYP3A65 and CKma in zebrafish exposed to different concentrations of DKAs (Fig. 4). Zebrafish livers from DKA-exposure groups showed an obvious elevation in mRNA level for $A H R R a$ and a remarkable down-regulation for CYP3A65. Transcription levels for CYP3A65 in the $60 \mathrm{mg} / \mathrm{L}$ DKA treatment were significantly lower $(p<0.05)$ than the control group. The CKma expression from skeletal muscle was significantly up-regulated $(p<0.05)$ in the $60 \mathrm{mg} / \mathrm{L}$ treatment, but down-regulated $(p<0.01)$ in the $90 \mathrm{mg} / \mathrm{L}$ treatment after two months of DKA exposure when compared to the control group.

\section{Histopathological analysis for liver and skeletal muscle}

Zebrafish at $60 \mathrm{dpf}$ were selected to investigate histopathological changes after exposure to DKAs $(0,45$,
60 and $90 \mathrm{mg} / \mathrm{L}$ ). Liver is a critical target for xenobiotics, and drug-induced liver injury is a major contributor in attrition of drugs in preclinical development. Although zebrafish liver does not show the obvious lobular structure as present in mammals, substructures such as bile canaliculi can be discerned. In the control group, hepatocytes have uniform nucleus, evenly distributed cytoplasm, distinct myelin-body (Fig. 5) and some lysosome. Most of mitochondria were round or oval and contained welldeveloped cristae. However in DKA-exposed groups, the ultrastructure changes were mainly to the mitochondria cristae, which were reduced, swollen and vague, and disrupted with chaotic distribution. The rough endoplasmic reticulum (RER) decreased or even disappeared, some of cells experienced edema and necrosis, and the lysosome was increased (Fig. 5). Meanwhile, intracellular and intra-canalicular cholestasis, partial dissolution of cell nuclear membranes, karyopyknosis, and chromatic agglutination were also observed. Moreover, lipid droplets, 
Toxicity of $\beta$-diketone antibiotics to zebrafish
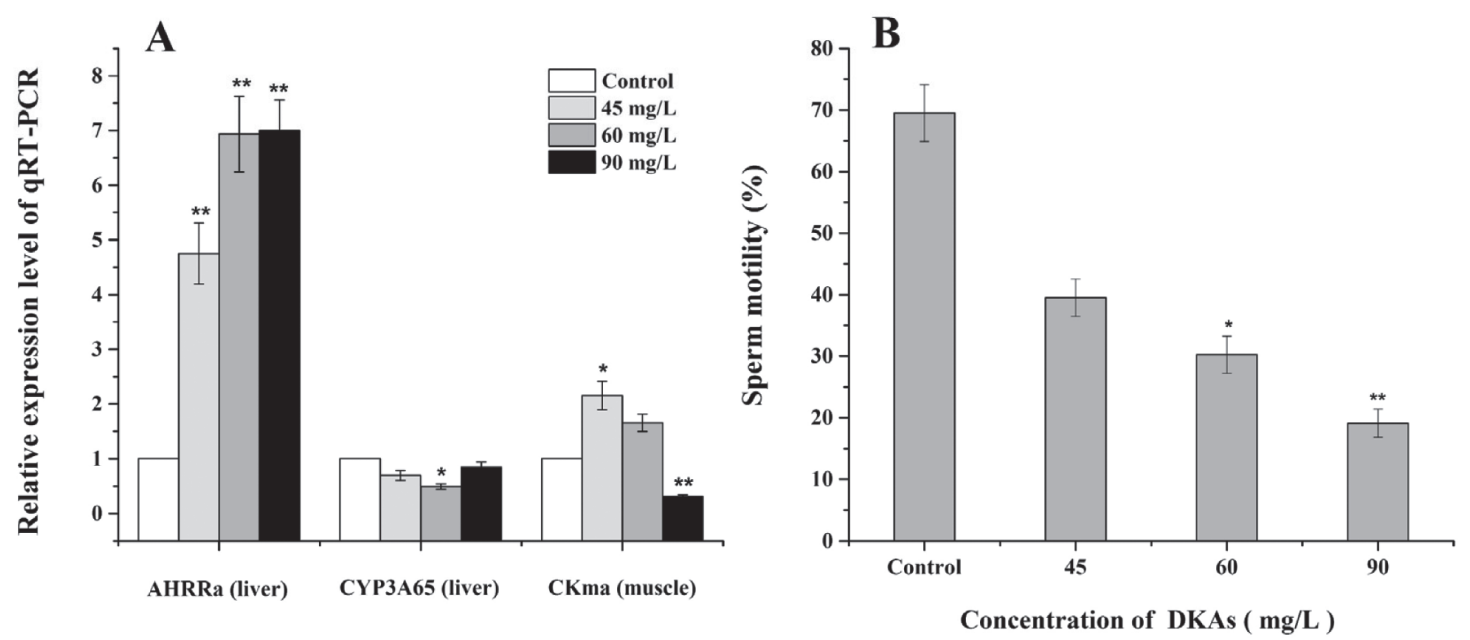

Fig. 4. Expression of candidate genes and sperm motility. (1) A, expression changes of candidate genes at transcriptional level by qRT-PCR; (2) B, effects of DKA exposure concentrations on sperm motility; (3) Determination in zebrafish liver and skeletal muscle after 2 months of DKA exposure at 0, 45, 60 or $90 \mathrm{mg} / \mathrm{L}$; (4) AhRRa and CYP3A65 expression in liver; (5) CKma expression in skeletal muscle; $(6) *$ and $* *$ indicate significant difference from control at $p<0.05$ and $p<0.01$ levels, respectively; (7) Values represent the average \pm S.D. $(n=3)$.

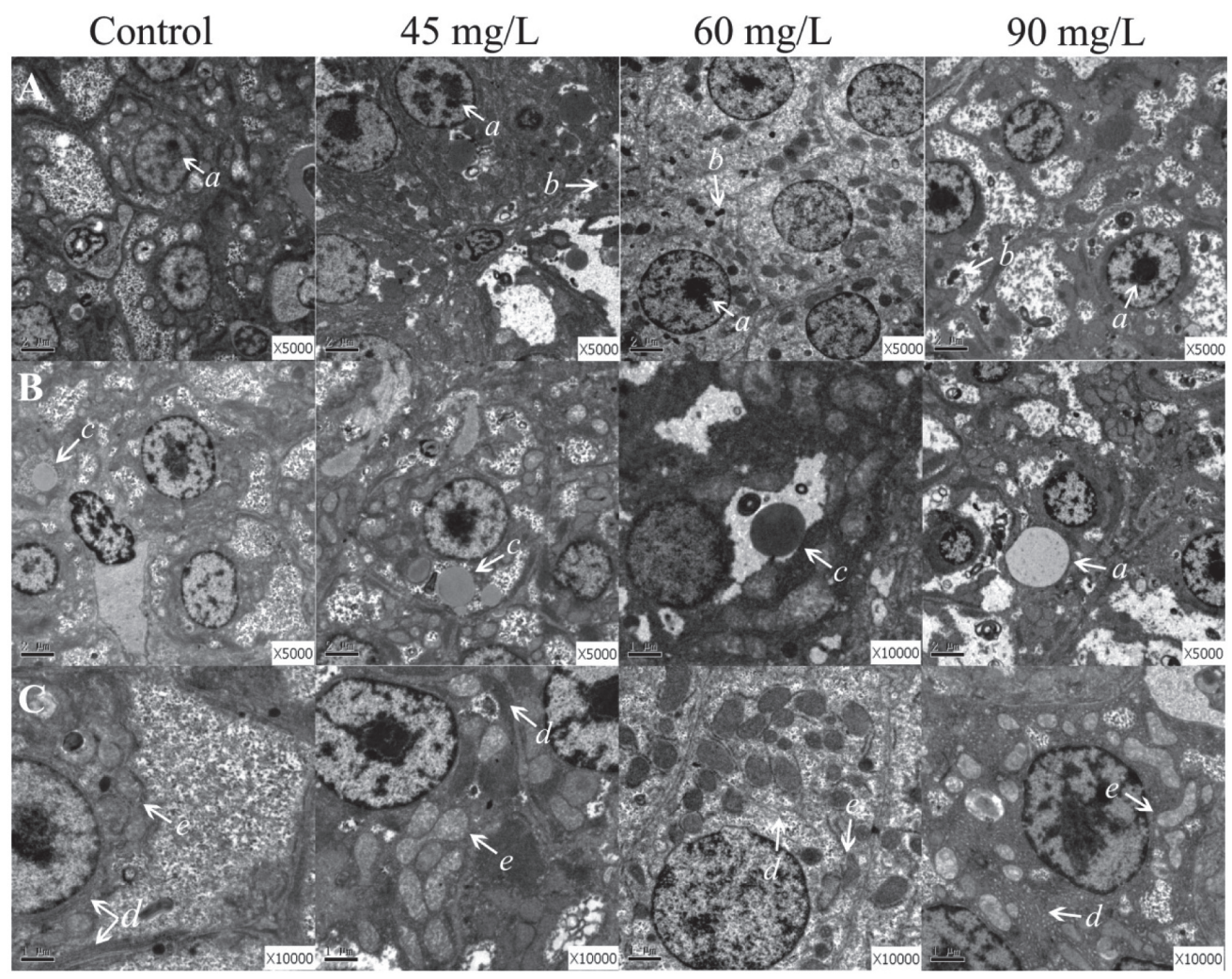

Fig. 5. TEM images for zebrafish liver. (1) A indicates karyopyknosis and cholestatis; (2) B indicates lipid droplet deposition in lipid particles; (3) C indicates endoplasmic reticulum and mitochondria; (4) a, karyopyknosis; (5) b, cholestatis; (6) c, lipid droplet deposition; (7) d, endoplasmic reticulum; (8) e, mitochondria. 
microvesicular steatosis and devour vesicles were found in some cytoplasm (Fig. 5). The severity of each abnormal phenomenon was dependent on DKA exposure concentration.

In comparison with the control group, DKA-exposed groups showed a narrow sarcostyle, and the surrounding sarcoplasmic reticulum hyperplasia, as well as defects in the sarcomeric assembly characterized by disorganized sarcomere structure with blurry Z-lines. Although DKAs did not obviously affect the length of the A-band and sarcomeres, the I-band was broadened, as well as the H-band, which is in the middle area of the A-band (Fig. 6A-D). In addition, there were necrotic areas and a large number of edema in the sarcoplasmic reticulum, and myofibrils were arranged disorderly with some disrupted or dissolved (Fig. 6E-H). As the myofibrils dissolved, part of the mitochondria gathered and was distributed in the sarcoplasmic reticulum with a disordered arrangement. Vacuolization or edema in organelles such as mitochondria in striated muscle cells were also observed in the DKA-exposed groups. Meanwhile, mitochondria swelling, reduction of mitochondrial cristae, and disruption of cristae with disordered arrangement were found in DKA-exposed groups (Fig. 6I-L).

\section{Sperm motility analysis}

Sperm motility has the potential to affect the reproductive ability of male zebrafish. Fish sperm is very sensitive to environmental pollutants, and thus can be used as one of the sensitive biomarkers to evaluate endocrine disrupting effects of some pollutants. A gradually decreasing trend of total sperm motility (TSM) was observed with increasing DKA concentrations (Fig. 4B). Zebrafish TSM was less than $40 \%$ in the $90 \mathrm{mg} / \mathrm{L} \mathrm{DKA} \mathrm{treatment,}$ which was a ca. $30 \%$ decrease as compared with the control group.

\section{DISCUSSION}

The reported environmental concentrations of sin-
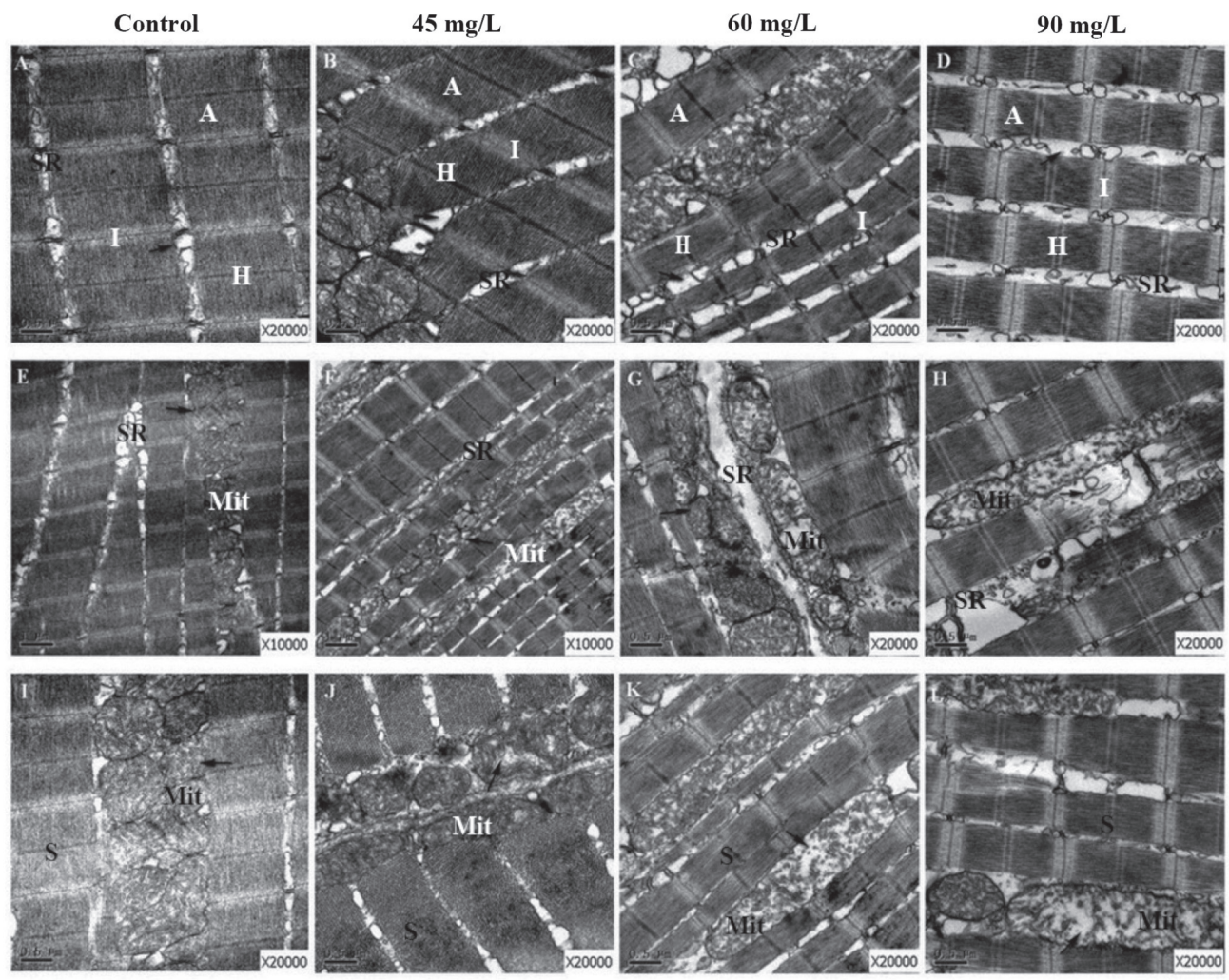

Fig. 6. TEM images for zebrafish skeletal muscles. (1) A, E and I indicate control; (2) B, F and J indicate 45 mg/L DKA exposure concentration; (3) C, G and $\mathrm{K}$ indicate $60 \mathrm{mg} / \mathrm{L}$ DKA exposure concentration; (4) D, H and L indicate $90 \mathrm{mg} / \mathrm{L}$ DKA exposure concentration; (5) "A", A-band; (5) "I", I-band; (6) "H" H-band; (7) "SR", sarcroplasmic reticulum; (8) "Mit", mitochontriosome; (9) "S", sarcromere; (10) A-D, widen and severe edema in I-band, A-band and sarcoplasmic reticulum; (11) $\mathrm{E}-\mathrm{H}$, melted muscle fiber and severe edema in sarcoplasmic reticulum; (12) I-L, crest ablation and edema in mitochondria. 
Toxicity of $\beta$-diketone antibiotics to zebrafish

gle DKA species had large differences in different source waters. Ashfaq et al. (2016) reported the highest concentrations of ofloxacin, ciprofloxacin, sparfloxacin and moxifloxacin were $66,18,58$ and $224 \mu \mathrm{g} / \mathrm{L}$, respectively, in two major hospitals of Lahore, Pakistan (Ashfaq et al., 2016). Apart from urban and hospital wastewaters, FQs enter the environment through bulk drug producing activities, with FQ wastewater concentrations ranging from $6 \mu \mathrm{g} / \mathrm{L}$ to $31 \mathrm{mg} / \mathrm{L}$. Average concentrations of ofloxacin, norfloxacin and ciprofloxacin in production facility wastewaters were $0.1,0.2$ and $4.9 \mathrm{mg} / \mathrm{L}$, respectively, and were generally higher than hospital and urban wastewater concentrations (Van Doorslacr et al., 2014). Average FQ concentrations in chicken, pig and cow manures from eight provinces in China ranged from 1 to $5 \mathrm{mg} / \mathrm{kg}$ (Zhao et al., 2010). FQs used in larvae aquaculture ponds during the shrimp development period, resulted in FQ pond water concentrations ranging from 0.3 to $1.2 \mathrm{mg} / \mathrm{L}$ (Thuy et al., 2011). The above-mentioned literature demonstrates that DKA residues may reach $\mathrm{mg} / \mathrm{L}$ levels in several environments. Based on the $\mathrm{LC}_{50}$ of the six DKAs utilized in this study, we selected $7.5,10$ and $15 \mathrm{mg} / \mathrm{L}$ as the exposure concentration for single DKA species (corresponding to 45, 60 and $90 \mathrm{mg} / \mathrm{L}$ for the DKA mixture) for acute toxicity testing.

Based on our previous research examining biomarkers and behavior of zebrafish, the concentration of the representative DKA species was selected as 0.39-6.25 mg/L (of each DKA species) for exposure to zebrafish. A highthroughput RNA sequencing technique was used to compare transcriptional data of zebrafish in control and DKAexposed treatment groups. We obtained differentially expressed genes, compared the transcription differences between control and treatment samples, and determined specific gene markers for correlation with drug-induced diseases. From these data, the molecular mechanisms for chronic toxicity effects and the ecological risk to DKA exposure were assessed (Wang et al., 2016a, 2016b). This study is related to acute toxicity testing of DKAs, which allows us an experimental approach to directly observe the toxicological effects resulting from DKA exposure to zebrafish.

To comprehensively determine the roles of various DKA-exposure treatments on the transcriptome of the zebrafish model, we performed whole-genome transcriptome sequencing on the Illumina Hi-Seq 2000 sequencing platform. Paired-end sequencing mode $(2 \times 100 \mathrm{bp})$ was employed to match more accurately the reference genome sequence and to improve sequencing efficiency. In this study, $8-10 \mathrm{G}$ of sequencing data were collected. The present sequencing data were completely saturat- ed and sufficient for subsequent analysis (Supplementary Table 4) (Wu et al., 2010). qPCR analysis was carried out to confirm the direction of change detected by digital gene expression analysis. To achieve an unbiased functional annotation of the differentially expressed genes that were identified by digital gene expression analysis, we tested for significant enrichment of Gene Ontology and pathways groups (Supplementary Table 5 and Supplementary Fig. 2, Fig.3).

Through KEGG analysis, pathway_id ko00250 and ko04530 were closely related to liver functions (Supplementary Table 5). Therefore, we determined the MDA concentration, the expressions of $A H R R a$ and $C Y P 3 A 65$, and TEM of zebrafish liver thin sections, which were concerned with liver functions. In addition, pathway id ko00970 was related to neurodegeneration (Lee et al., 2006) and our previous study also found that DKAs had significant effects on zebrafish locomotion behavior in both light and light-to-dark photoperiods (Wang et al., 2014). As a result, we determined the endpoints on locomotion behavior, e.g. $\mathrm{CK}$ and $\mathrm{AChE}$ activities, $\mathrm{Cr}$ concentration, CKma expression, and TEM of skeletal muscle. In the future, a deep investigation is required to analyze digital gene expressions and to explore the relationship between functions of these differential genes and the changes of endpoints observed in this study.

Zebrafish possess duplicate aryl hydrocarbon receptor (AHR)-repressor AHRR paralogs that act in a negative feedback loop to repress the AHR signaling pathway (Evans et al., 2005). AHR-response element sequences are present in the promoters of many teleost CYP3 genes, but not of mammalian CYP3 genes, suggesting that AHR/ AHR2-mediated transcription is likely a common regulatory mechanism for teleost CYP3 genes (Chang et al., 2013). AHRR is involved in human reproductive physiology and in the regulation of cell growth (Hahn et al., 2009). Jenny and co-workers hypothesized that $A H R R$ may play a role in basic biological processes, in addition to its role in toxicology (Jenny et al., 2009). It has been estimated that over $75 \%$ of currently used drugs are metabolized by CYP3As (Guengerich, 2008). Orthologous $C Y P 3 A$ genes are also found in other non-mammalian organisms, including chickens, amphibians and fish. Tseng and co-workers identified CYP3A65 in zebrafish, a CYP3A ortholog that is constitutively expressed in liver and intestinal tissues (Tseng et al., 2005). Further, they observed that reducing translation of either PXR or AHR2 dramatically reduced endogenous transcription of CYP3A65. In this investigation, hepatic expression of a number of AHR-regulated genes was studied to investigate if DKAs can activate gene transcription through the 
AHR pathway in zebrafish. DKA exposure down-regulated the expression of $C Y P 3 A 65$, but up-regulated the mRNA levels of AHRRa, consistent with the findings of Tseng and co-workers (Tseng et al., 2005).

Zebrafish and mammals have a high similarity in $\mathrm{Cr}$ synthesis and transport mechanisms. The $\mathrm{Cr} / \mathrm{PCr} / \mathrm{CK}$ system is one of the most important mechanisms supplying energy in vertebrates. Typically, both internal and external environmental alterations in an organism can cause behavioral changes, which often reflects the interaction of an animal with its environment (Jenny et al., 2009). Indeed, when an animal is exposed to environmental contaminants, behavioral alteration is often the first defensive response (Bégout and Lagardere, 2004). Zebrafish locomotor behavior is a direct result of nerve conduction, muscle contraction and energy transfer. Our previous studies demonstrated that DKAs had an obvious effect on locomotor activity and pattern of zebrafish response to photoperiod stimulation (Wang et al., 2014).

The most sensitive embryotoxicological endpoint of DKAs was the impairment of central nervous system, leading to the smaller zebrafish tritocerebrum and the curvature of the notochord (Fig. 2). The previously behavioral study by our group revealed that DKAs reduced the swimming activity and induced equilibrium loss in larvae (Zhang et al., 2016). Another sensitive endpoint was the abnormal yolk sac resorption in conjunction with prominent oedemas. Frequently, these two abnormalities appear together in disturbed embryos, as stated by Oliveira et al. (2017). Their occurrence indicates metabolic disturbances in the embryo that prevent the normal use of stored energy in the yolk and cause abnormal accumulations of fluid in the pericardial cavity (Miyares et al., 2014). Also, a series of results in this investigation elucidated the abovementioned adverse effects of DKAs, which are related to the effects of DKAs on energy metabolism and transfer, TEM histopathological observation of adult muscle tissues (especially muscle fiber ablation and swelling of mitochondria), and transcriptional changes in CKma gene. Since the successful aquatic adaptation of zebrafish larvae is dependent on swimming coordination, closure of swimming bladder plays an important role in swimming coordination. Under environmental stress, zebrafish is marked by the opening and closure of swimming bladder, and thus it is a good indicator of the reduced fitness caused by a specific pollutant (Braunbeck et al., 2015).

There are a number of motor neurons in the spinal cord, dominating skeletal muscle cells. Motor neurons not only regulate the contraction of target muscle, but also have an important neurotrophic effect on muscles. The cut-off of motor nerves can result in a series of pathological chang- es such as quick protein decomposition, muscle atrophy, etc. In this investigation, TEM observations on spinal cord proved that a series of neuron pathological changes occurred such as axonal demyelination. Correspondingly, a large number of lesions occurred in muscular cells (Fig. 6), which was consistent with the decreasing of muscular neurotropic effects. These results suggested that DKAs exposure might affect zebrafish movement nervous system, resulting in the reduced or disappeared neurotropic effects and further activities of down muscular group.

Mitochondria are the important sites of ATP hydrolysis energy supply, which can provide energy for metabolic activities. By TEM observation, we found that the mitochondria in many kinds of tissues such as liver and muscle appear abnormal phenotypes due to DKAs exposure. In order to further verify the above phenomenon, we selected sperm cells for further study. There are abundant mitochondria in the tail of sperm cells, which can provide energy for sperm swimming. After DKAs exposure, zebrafish sperm motility was significantly decreased with increasing in exposure concentrations, which was in agreement with our observation in TEM. These results proved that DKAs exposure led to severe damage to mitochondria. Mitochondria play a critical role in breaking down food sources and thus any flaws in the mitochondria can have a serious impact on organism health. Fent and co-workers found that mitochondria damage from chemicals caused edema due to interference with metabolism or osmotic pressure regulation (Fent and Meier, 1992). In hepatocytes from the DKA-exposure groups, karyopyknosis, agglutination or frontier set of chromatin, deepening of mitochondrial cristae bands and chaotic distribution were observed. Similarly, Amacher and Martin showed that the canine hepatocyte is susceptible to tetracycline-induced steatosis, when TG accumulation was concomitant with the inhibition of mitochondrial lipid metabolism (Amacher and Martin, 1997). In addition, microvesicular steatosis and devour vesicles were observed in some cytoplasms. Tetracycline belongs to a group of drugs known to induce microvesicular steatosis (Yin et al., 2006). The functions of the endoplasmic reticulum are closely related to synthesis, detoxification, and assimilation of carbohydrates and lipids, as well as transport proteins. Endoplasmic reticulum swelling in hepatocytes was observed under DKA exposure, demonstrating that DKAs may inhibit detoxification functions of hepatocytes, and that subsequent damage to the cell nucleus may result in genetic toxicity. Consequently, this work provides insights to explore the discovery of new potential diagnostic biomarkers and drug targets for hepatosis caused by DKAs. 
Toxicity of $\beta$-diketone antibiotics to zebrafish

\section{ACKNOWLEDGMENTS}

This work is jointly supported by the National Natural Science Foundation of China (31270548 and 21577107), the Natural Science Foundation of Zhejiang Province (LY15C030004 and LY15B070009), and the Public Beneficial Project of Wenzhou City Sci \& Technol Bureau (H20150005). The authors thank Professor Randy A. Dahlgren, employed in Davis, University of California, USA, for his correction of this manuscript.

Conflict of interest---- The authors declare that there is no conflict of interest.

\section{REFERENCES}

Allen, J.P. and Neely, M.N. (2010): Trolling for the ideal model host: Zebrafish take the bait. Future Microbiol., 5, 563-569.

Amacher, D.E. and Martin, B.A. (1997): Tetracycline-induced steatosis in primary canine hepatocyte cultures. Fundam. Appl. Toxicol., 40, 256-263.

Ashfaq, M., Khan, K.N., Rasool, S., Mustafa, G., Nazar, M.F., Sun, Q. and Yu, C.P. (2016): Occurrence and ecological risk assessment of fluoroquinolone antibiotics in hospital waste of Lahore, Pakistan. Environ. Toxicol. Pharmacol., 42, 16-22.

Bégout, A.M.L. and Lagardére, J.P. (2004): Measuring cultured fish swimming behaviour: First results on rainbow trout using acoustic telemetry in tanks. Aquaculture, 240, 175-186.

Ben, W., Qiang, Z., Adams, C., Zhang, H. and Chen, L. (2008) : Simultaneous determination of sulfonamides, tetracyclines and tiamulin in swine wastewater by solid-phase extraction and liquid chromatography-mass spectrometry. J. Chromatogr. A, 1202, 173-180.

Bondad-Reantaso, M.G., Arthur, J.R. and Subasinghe, R.P. (2012): Improving biosecurity through prudent and responsible use of veterinary medicines in aquatic food production. In: FAO (Ed.), Fisheries and Aquaculture Technical Paper. Food and Agriculture Organisation of the United Nations, Rome, pp., 207.

Braunbeck, T., Kais, B., Lammer, E., Otte, J., Schneider, K., Stengel, D. and Strecker, R. (2015): The fish embryo test (FET): origin, applications, and future. Environ. Sci. Pollut. Res., 22, 16247-16261.

Chang, C.T., Chung, H.Y., Su, H.T., Tseng, H.P., Tzou, W.S. and Hu, C.H. (2013): Regulation of zebrafish CYP3A65 transcription by AHR2. Toxicol. Appl. Pharmacol., 270, 174-184.

Craig, M.P., Gilday, S.D. and Hove, J.R. (2006): Dose-dependent effects of chemical immobilization on the heart rate of embryonic zebrafish. Lab. Anim., 35, 41-47.

Dong, L., Gao J., Xie, X. and Zhou, Q. (2012): DNA damage and biochemical toxicity of antibiotics in soil on the earthworm Eisenia fetida. Chemosphere, 89, 44-51.

Evans, B.R., Karchner, S.I., Franks, D.G. and Hahn, M.E. (2005): Duplicate aryl hydrocarbon receptor repressor genes (ahrrl and ahrr2) in the zebrafish Danio rerio: Structure, function, evolution, and AHR-dependent regulation in vivo. Arch. Biochem. Biophys., 441, 151-167.

Fent, K. and Meier, W. (1992): Tributyltin-induced effects on early life stages of minnows Phoxinus phoxinus. Arch. Environ.
Contam. Toxicol., 22, 428-438.

Guengerich, F.P. (2008): Cytochrome P450 and chemical toxicology. Chem. Res. Toxicol., 21, 70-83.

Hahn, M.E., Allan, L.L. and Sherr, D.H. (2009): Regulation of constitutive and inducible AHR signaling: Complex interactions involving the AHR repressor. Biochem. Pharmacol., 77, 485497.

Jansen, K.L., Cole, T.B., Park, S.S., Furlong, C.E. and Costa, L.G. (2009): Paraoxonase 1 (PON1) modulates the toxicity of mixed organophosphorus compounds. Toxicol. Appl. Pharmacol., 236, 142-153.

Jenny, M.J., Karchner, S.I., Franks, D.G., Woodin, B.R., Stegeman, J.J. and Hahn, M.E. (2009): Distinct roles of two zebrafish AHR repressors (AHRRa and AHRRb) in embryonic development and regulating the response to 2,3,7,8-tetrachlorodibenzo- $p$-dioxin. Toxicol. Sci., 110, 426-441.

Jones, O.A.H., Voulvoulis, N. and Lester, J.N. (2002): Aquatic environmental assessment of the top 25 English prescription pharmaceuticals. Water Res., 36, 5013-5022.

Karci, A. and Balcioğlu, I.A. (2009): Investigation of the tetracycline, sulfonamide, and fluoroquinolone antimicrobial compounds in animal manure and agricultural soils in Turkey. Sci. Total Environ., 407, 4652-4664.

Khadra, A., Pinelli, E., Lacroix, M.Z., Bousquet-Melou, A., Hamdi, H., Merlina, G., Guiresse, M. and Hafidi, M. (2012): Assessment of the genotoxicity of quinolone and fluoroquinolones contaminated soil with the Vicia faba micronucleus test. Ecotoxicol. Environ. Saf., 76, 187-192.

Lanza, F. and Sellergren, B. (1999): Method for synthesis and screening of large groups of molecularly imprinted polymers. Anal. Chem., 71, 2092-2096.

Lee, J.W., Beebe, K., Nangle, L.A., Jang, J., Longo-Guess, C.M., Cook, S.A., Davisson, M.T., Sundberg, J.P., Schimmel, P. and Ackerman, S.L. (2006): Editing-defective tRNA synthetase causes protein misfolding and neurodegeneration. Nature, 443, 50-55.

Lee, Y.J., Lee, S.E., Lee, D.S. and Kim, Y.H. (2008): Risk assessment of human antibiotics in Korean aquatic environment. Environ. Toxicol. Pharmacol., 26, 216-221.

Li, Q., Peng, S., Sheng, Z. and Wang, Y. (2010): Ofloxacin induces oxidative damage to joint chondrocytes of juvenile rabbits: Excessive production of reactive oxygen species, lipid peroxidation and DNA damage. Eur. J. Pharmacol., 626, 146-153.

Li, X.T., Chen, R., Jin, L.M. and Chen, H.Y. (2009): Regulation on energy metabolism and protection on mitochondria of Panax ginseng polysaccharide. Am. J. Chin. Med., 37, 1139-1152.

Lindberg, R., Jarnheimer, P.A., Olsen, B., Johansson, M., Tysklind, M. (2004): Determination of antibiotic substances in hospital sewage water using solid phase extraction and liquid chromatography/mass spectrometry and group analogue internal standards. Chemosphere, 57, 1479-1488.

Martyniuk, C.J., Gerrie, E.R., Popesku, J.T., Ekker, M. and Trudeau, V.L. (2007): Microarray analysis in the zebrafish (Danio rerio) liver and telencephalon after exposure to low concentration of 17alpha-ethinylestradiol. Aquat. Toxicol., 84, 38-49.

Miyares, R.L., de Rezende, V.B. and Farber, S.A. (2014): Zebrafish yolk lipid processing: a tractable tool for the study of vertebrate lipid transport and metabolism. Dis. Model. Mech., 7, 915-927.

Oliveira, J., Galhano, V., Henriques, I., Soares, A. and Loureiro, S. (2017): Basagran ${ }^{\circledR}$ induces developmental malformations and changes the bacterial community of zebrafish embryos. Environ. Pollut., 221, 52-63. 
Qu, S., Wang, X., Tong, C. and Wu, J. (2010): Metal ion mediated molecularly imprinted polymer for selective capturing antibiotics containing beta-diketone structure. J. Chromatogr. A, 1217, 8205-8211.

Rico, A., Satapornvanit, K., Haque, M.M., Min, J., Nguyen, P.T., Telfer, T.C. and van den Brink, P.J. (2012): Use of chemicals and biological products in Asian aquaculture and their potential environmental risks: A critical review. Rev. Aquacult., 4, 75-93.

Schwartz, J., Holmuhamedov, E., Zhang, X., Lovelace, G.L., Smith, C.D. and Lemasters, J.J. (2013): Minocycline and doxycycline, but not other tetracycline-derived compounds, protect liver cells from chemical hypoxia and ischemia/reperfusion injury by inhibition of the mitochondrial calcium uniporter. Toxicol. Appl. Pharmacol., 273, 172-179.

Scott, G.R. and Sloman, K.A. (2004): The effects of environmental pollutants on complex fish behaviour: Integrating behavioural and physiological indicators of toxicity. Aquat. Toxicol., 68, 369-392.

Sendzik, J., Shakibaei, M., Schäfer-Korting, M., Lode, H. and Stahlmann, R. (2010): Synergistic effects of dexamethasone and quinolones on human-derived tendon cells. Int. J. Antimicrob. Agents., 35, 366-374.

Sheng, Z.G., Huang, W., Liu, Y.X., Yuan, Y. and Zhu, B.Z. (2013): Ofloxacin induces apoptosis via $\beta 1$ integrin-EGFR-Rac1-Nox2 pathway in microencapsulated chondrocytes. Toxicol. Appl. Pharmacol., 267, 74-87.

Shi, Y., Gao, L., Li, W., Liu, J. and Cai, Y. (2012): Investigation of fluoroquinolones, sulfonamides and macrolides in longterm wastewater irrigation soil in Tianjin, China. Bull. Environ. Contam. Toxicol., 89, 857-861.

Sipes, N.S., Padilla, S. and Knudsen, T.B. (2011): Zebrafish: as an integrative model for twenty-first century toxicity testing. Birth. Defects Res. Part. C, 93, 256-267.

Smith-Ryan, A.E., Ryan, E.D., Fukuda, D.H., Costa, P.B., Cramer, J.T. and Stout, J.R. (2014): The effect of creatine loading on neuromuscular fatigue in women. Med. Sci. Sports Exerc., 46, 990-997.

Thuy, H.T.T., Nga le, P. and Loan, T.T. (2011): Antibiotic contaminants in coastal wetlands from Vietnamese shrimp farming. Environ. Sci. Pollut. Res., 18, 835-841.

Tseng, H.P., Hseu, T.H., Buhler, D.R., Wang, W.D. and Hu, C.H. (2005) : Constitutive and xenobiotics-induced expression of a novel CYP3A gene from zebrafish larva. Toxicol. Appl. Pharmacol., 205, 247-258.

Van Doorslaer, X., Dewulf, J., Van Langenhove, H. and Demeestere, K. (2014): Fluoroquinolone antibiotics: An emerging class of environmental micropollutants. Sci. Total. Environ., 500-501, 250-269.

Wang, H.L., Che, B.G., Duan, A.L., Mao, J.W., Dahlgren, R.A.,
Zhang, M.H., Zhang, H.Q., Zeng, A.B. and Wang, X.D. (2014): Toxicity evaluation of $\beta$-diketone antibiotics on the development of embryo-larval zebrafish (Danio rerio). Environ. Toxicol., 29, 1134-1146.

Wang, H.L., Yin, X.H., Li, F.H., Dahlgren, R.A., Zhang, Y.N., Zhang, H.Q. and Wang, X.D. (2016a): Chronic toxicological effects of $\beta$-diketone antibiotics on zebrafish (Danio rerio) using transcriptome profiling of deep sequencing. Environ. Toxicol., 31, 1357-1371.

Wang, X.D., Zheng, Y.S., Zhang, Y.N., Li, J.Y., Zhang, H.Q. and Wang, H.L. (2016b): Effects of $\beta$-diketone antibiotic mixtures on behavior of zebrafish (Danio rerio). Chemosphere, 144, 21952205.

Westerfield, M. (2000): The zebrafish book. Guide for the laboratory use of zebrafish (Danio rerio). Eugene. University of Oregon Press.

Wu, J.Q., Habegger, L., Noisa, P., Szekely, A., Qiu C., Hutchison, S., Raha, D., Egholm, M., Lin, H., Weissman, S., Cui, W., Gerstein, M. and Snyder, M. (2010): Dynamic transcriptomes during neural differentiation of human embryonic stem cells revealed by short, long, and paired-end sequencing. Proc. Nat. Acad. Sci. USA, 107, 5254-5259.

Xie, X.J., Zhou, Q.X., Bao, Q.H., He, Z. and Bao, Y.Y. (2011): Genotoxicity of tetracycline as an emerging pollutant on root meristem cells of wheat (Triticum aestivum L.). Environ. Toxicol., 26, 417-423.

Yang, G.F., Zhang, Q.Q. and Jin, R.C. (2013): Changes in the nitrogen removal performance and the properties of granular sludge in an Anammox system under oxytetracycline (OTC) stress. Bioresour. Technol., 129, 65-71.

Ye, L.B., Zhang, J.S., Ye, X., Tang, Q., Liu, Y., Gong, C., Du, X. and Pan, Y. (2008): Structural elucidation of the polysaccharide moiety of a glycopeptide (GLPCW-II) from Ganoderma lucidum fruiting bodies. Carbohydr. Res., 343, 746-752.

Yin, H.Q., Kim, M., Kim, J.H., Kong, G., Lee, M.O., Kang, K.S., Yoon, B.I., Kim, H.L. and Lee, B.H. (2006): Hepatic gene expression profiling and lipid homeostasis in mice exposed to steatogenic drug, tetracycline. Toxicol. Sci., 94, 206-216.

Zhang, H., Luo, Y. and Zhou, Q.X. (2008): Research advancement of ecotoxicity of chlortetracycline antibiotics. J. Agro-Environ. Sci., 27, 407-413.

Zhao, L., Dong, Y.H. and Wang, H. (2010): Residues of veterinary antibiotics in manures from feedlot livestock in eight provinces of China. Sci. Total. Environ., 408, 1069-1075.

Zhang, Y.N., Wang, X.D., Yin, X.H., Shi, M.R., Dahlgren, R.A. and Wang, H.L. (2016): Toxicity assessment of combined fluoroquinolone and tetracycline exposure in zebrafish (Danio rerio). Environ. Toxicol., 31, 736-750. 